\title{
The dedicated QEXAFS facility at the SLS: Performance and Scientific Opportunities
}

\author{
R. Frahm ${ }^{\mathrm{a}}$, M. Nachtegaal ${ }^{\mathrm{b}}$, J. Stötzel ${ }^{\mathrm{a}}$, M. Harfouche ${ }^{\mathrm{b}}$, \\ J.A. van Bokhoven ${ }^{\mathrm{c}}$ and J.-D. Grunwaldt ${ }^{\mathrm{d}}$ \\ ${ }^{a}$ Dept. of Physics, Univ. Wuppertal, Wuppertal, Germany \\ ${ }^{b}$ Paul Scherrer Institut, Villigen, Switzerland \\ ${ }^{c}$ ETH Zurich, Institute of Chemical and Bioengineering, Zurich, Switzerland \\ ${ }^{d}$ Dept. of Chemical and Biochemical Engineering, Technical Univ. of Denmark, Lyngby, \\ Denmark
}

\begin{abstract}
The SuperXAS beamline at the Swiss Light Source (SLS) features a permanently installed monochromator for quick scanning EXAFS (QEXAFS) spectroscopy in series with a conventional double crystal monochromator (DCM). All installed optical components like collimating and focussing mirrors can be used by both devices. The remote exchange of the monochromators is possible in less than five minutes while maintaining the beam geometry on the sample. The QEXAFS system allows fast absorption scans down to the millisecond range for the investigation of time dependent processes. Using a $\mathrm{Si}(111)$ channel cut crystal the energy range from $5-16 \mathrm{keV}$ can be covered, with a $\mathrm{Si}(311)$ cut the range $9.5-30 \mathrm{keV}$. Usually a quick scanning interval of $0.1^{\circ}-2^{\circ}$ in Bragg angle is selected, thus covering XANES, full EXAFS or multiple edge scans of e.g. all L-edges of a heavy element. Up to about 80 spectra per second can be collected, corresponding to a time resolution of $12.5 \mathrm{~ms}$. The high intensity of the beamline even facilitates fluorescence measurements on dilute samples.
\end{abstract}

Keywords: X-ray absorption spectroscopy, XANES, EXAFS, QEXAFS, catalysis, time dependent studies.

PACS: 61.05.cj 07.85.Qe, 61.10.Ht, 82.45

\section{INTRODUCTION}

The QEXAFS technique is based on fast sequential measurements of the absorption coefficient "on-the-fly". Due to the high intensity at synchrotron radiation beamlines it is possible to measure full EXAFS spectra in a few seconds, if extremely stable conventional double crystal monochromator systems are used in combination with fast detection devices $[1,2]$. Faster data collection down to the ms-range requires special setups which continuously oscillate the monochromator crystals at high speed in Bragg angle. Out of stability reasons usually channel cut crystals with small channel width of typically $10 \mathrm{~mm}$ are used. For detection of the photon intensity before and after a transmission sample commonly ionization chambers or photodiodes connected to current amplifiers are employed. The output signals are directly digitized by fast analogue to digital converters and processed in a PC. The currently most advanced QEXAFS monochromator development is based on an eccentric disc, which is rotated by a motor and thus creates an oscillating movement of the crystal mount $[3,4]$. This monochromator concept was tested at several synchrotron radiation sources ranging from $1^{\text {st }}$ to $3^{\text {rd }}$ generation and is the basis for the design at the SLS, where a commercial version was installed [5]. In addition, an angular encoder allows the exact determination of the Bragg angle and thus energy scale of the measurements even at high speeds [6].

The QEXAFS method has been successfully applied to a wide variety of scientific investigations such as surface studies, solid state and chemical reactions, electrochemistry and catalysis research, which remains to be one of the main research areas [7].

CP1234, The10 ${ }^{\text {th }}$ International Conference on Synchrotron Radiation Instrumentation R. Garrett, I. Gentle, K. Nugent, and S. Wilkins

(c) 2010 American Institute of Physics 978-0-7354-0782-4/10/\$30.00 


\section{BEAMLINE OVERVIEW}

The basic layout of the SuperXAS Beamline at the SLS is given in Fig. 1. The QEXAFS Monochromator is installed in row with a conventional DCM. This allows usage of all installed optical components like collimating and focussing mirrors by both devices, which can be exchanged by remote control within less than 5 min. The QEXAFS monochromator allows spectra acquisition with an oscillation speed of up to about $40 \mathrm{~Hz}$ (equivalent to 80 spectra per second) and a scan range of up to $3 \mathrm{keV}$, depending on the selected edge energy and the eccentric used in the monochromator.

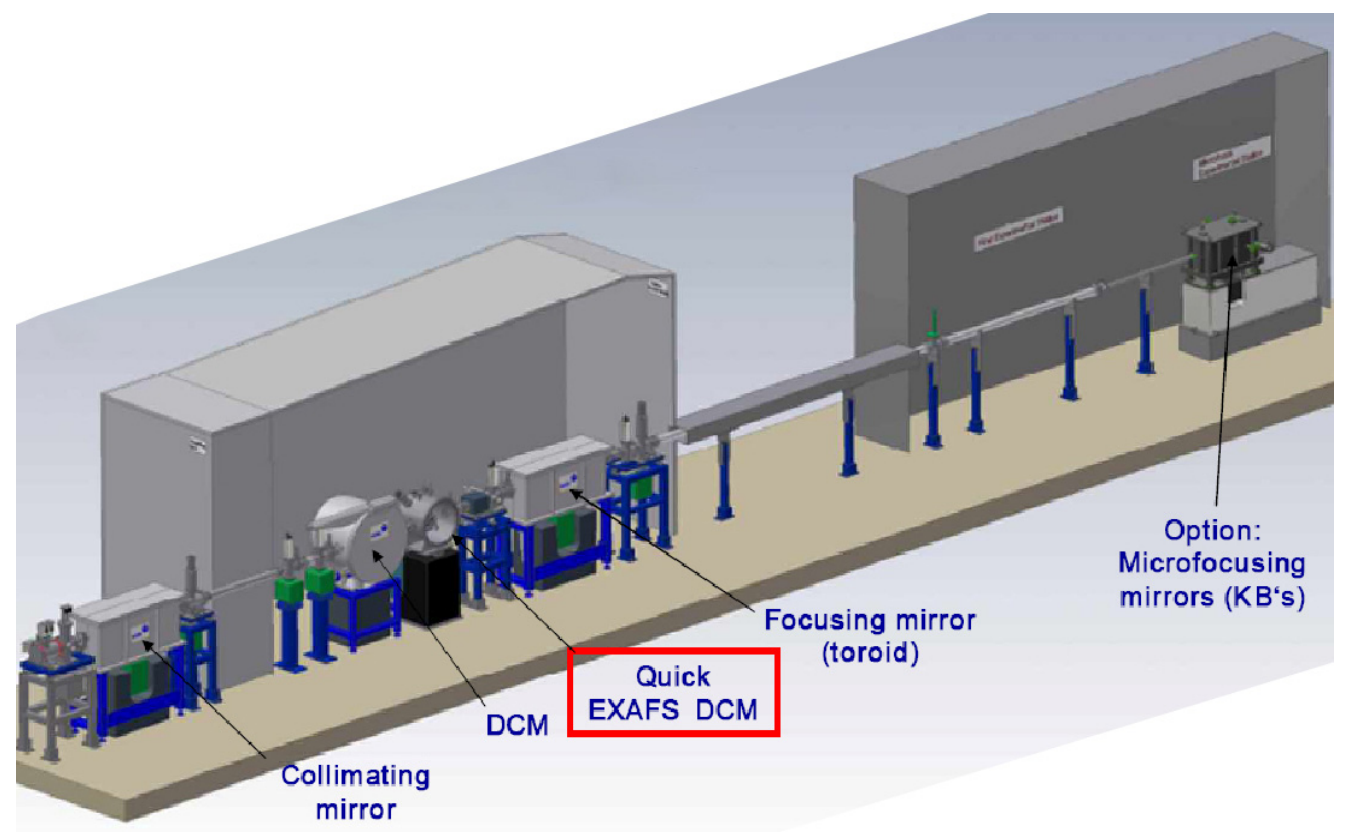

FIGURE 1. Layout of the SuperXAS Beamline at the SLS installed by Bruker ASC. The white beam enters from the left.

Many absorption spectra of reference metal foils were collected in transmission mode during the commissioning of the QEXAFS monochromator. For real samples, three ionization chambers can be read out simultaneously, resulting in the absorption spectra of the sample and of a reference compound, which usually is a metal foil. The monochromator has a high reproducibility over periods of several hours, but it is of advantage to have an additional reference signal available enabling the unambiguous detection of even small edge shifts. Ionization chambers were used as detectors. A photo of a typical experimental setup for catalysis research is given in Fig. 2. A small microreactor or a thin walled capillary with the catalyst powder is mounted on an adjustable stage between the first two ionization chambers. The connections to the gas supply are visible; the outgoing gas composition can be monitored with a mass spectrometer, which e.g. allows determining the catalytic activity. The SLS storage ring is usually operated at $2.4 \mathrm{GeV}$ and $400 \mathrm{~mA}$ electron current in top-up mode, resulting in a monochromatic photon flux of typically $10^{12}$ photons/s when a $\mathrm{Si}(111)$ crystal is used. The QEXAFS monochromator was equipped with either a $\mathrm{Si}(111)$ or a $\mathrm{Si}(311)$ channel cut crystal, which were usually oscillated with frequencies of 2 to $10 \mathrm{~Hz}$. Figures 3 and 4 show reference spectra obtained in the lower and higher energy range using the different crystals mentioned above. Those spectra illustrate the high data quality which is obtained over a wide energy range.

After an extremely short commissioning phase of less than two days after first beam on the QEXAFS monochromator the new equipment went into user operation in March 2008. Especially because the QEXAFS method does not require special efforts in sample preparation besides those necessary for standard XAFS experiments, the use of the whole setup is very user friendly. The demand for beamtime on this special device grew continuously, and currently about $30-50 \%$ of the total available beamtime at SuperXAS is used for research using the QEXAFS method. Main applications so far were in catalysis research (see e.g. [8,9,12]), and thus an example is given in the next section. 


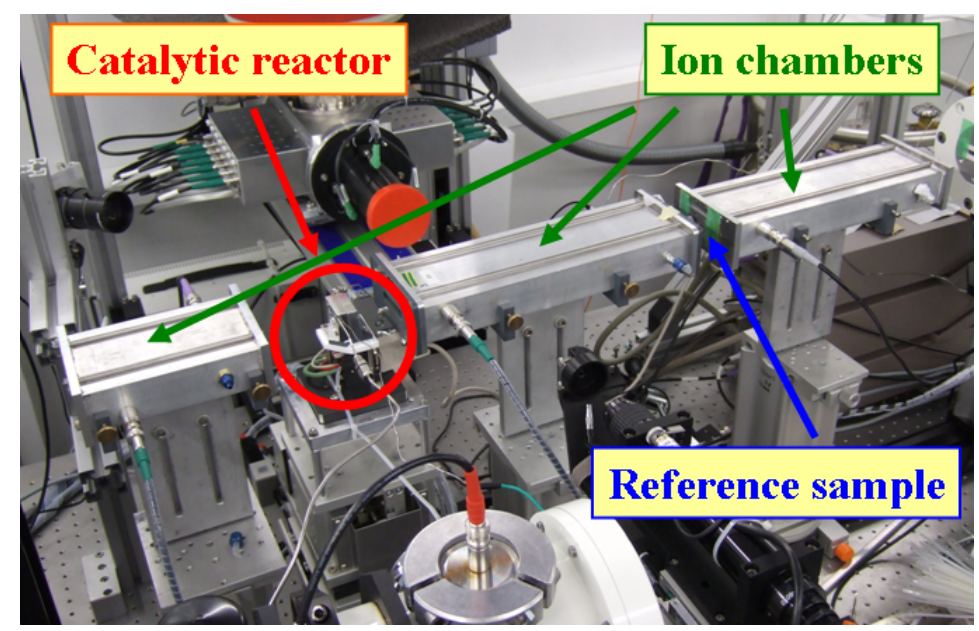

FIGURE 2. Experiment for catalysis research employing a heatable catalytic reactor. The monochromatized beam from the QEXAFS monochromator enters from the left.

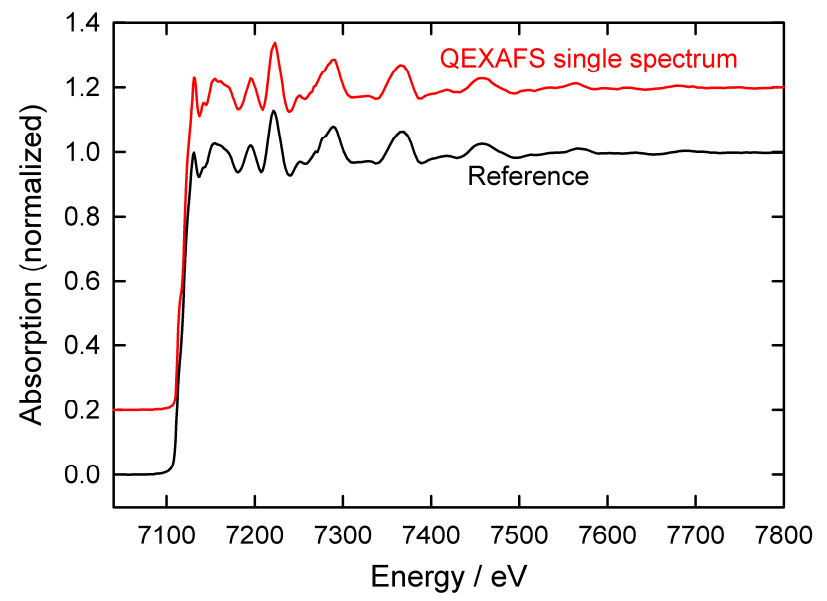

FIGURE 3. QEXAFS spectrum of a Fe-foil measured in $250 \mathrm{~ms}$ in comparison to a reference spectrum measured with a conventional monochromator. Both spectra were obtained using $\operatorname{Si}(111)$ crystals.

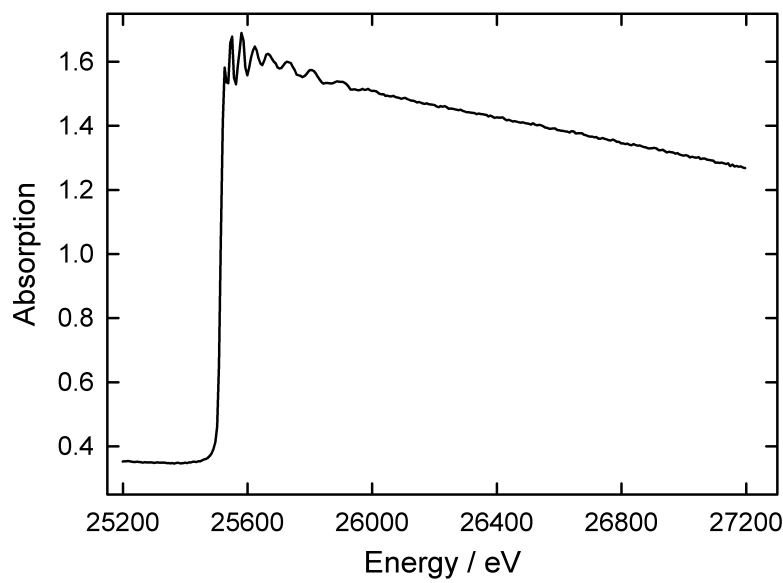

FIGURE 4. QEXAFS spectrum of an Ag-foil, measured with $1 \mathrm{~Hz}$ oscillation frequency using a Si(311) monochromator crystal, averaged over 18 spectra. 


\section{QEXAFS FOR CATALYSIS RESEARCH}

Figure 5 shows an example from catalysis research. Copper-based catalysts play an important role in methanol synthesis, steam reforming and for gas phase oxychlorination. QEXAFS studies in $\mathrm{Cu} / \mathrm{ZnO}$ systems have been reported in detail beforehand and indicate that the $\mathrm{Cu}(\mathrm{I})$ species are very short living intermediates $[10,11]$. In those cases XANES spectra were taken with subsecond timescale. Here, we extended the studies to $\mathrm{Cu} / \mathrm{Al}_{2} \mathrm{O}_{3}$ and full EXAFS data recording [12] and monitored the reduction in $5 \% \mathrm{H}_{2} / \mathrm{He}$ and re-oxidation in $21 \% \mathrm{O}_{2} / \mathrm{He}$ in a capillary microreactor. With an eccentric disk yielding $0.30^{\circ}$ angular range an energy range between about $8960 \mathrm{eV}$ and $9440 \mathrm{eV}$ was covered. Using a crystal oscillation frequency of $10 \mathrm{~Hz}$, this resulted in one spectrum per $50 \mathrm{~ms}$. As can be seen in Figure 5, this time resolution was required to properly monitor the re-oxidation, which occurred in less than a second, whereas the reduction occurred within several seconds. By cycling the reduction and reoxidation, further improvement of the spectra could be achieved [12].
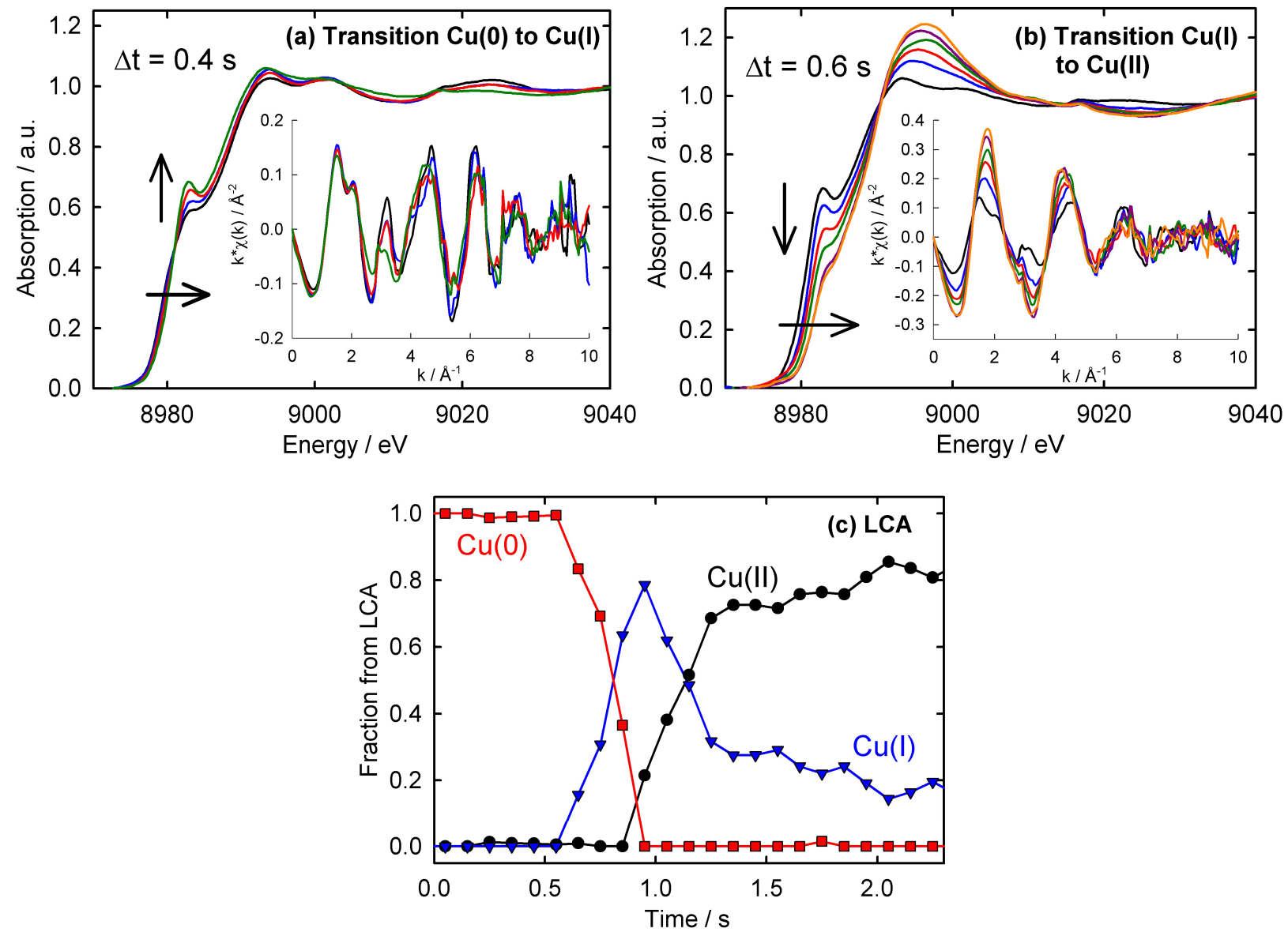

FIGURE 5. Re-oxidation of fully impregnated $\mathrm{Cu} / \mathrm{A}_{2} \mathrm{O}_{3}$ at $350^{\circ} \mathrm{C}$ in $21 \% \mathrm{O}_{2} / \mathrm{He}$ separated in two transition steps with $\chi(\mathrm{k})$-functions as insets. Linear combination analysis (LCA) shows that first $\mathrm{Cu}(\mathrm{I})$ and then $\mathrm{Cu}(\mathrm{II})$ are formed.

Another example for catalysis research recently investigated at the SuperXAS beamline was the ignition and extinction of the catalytic partial oxidation of methane over Pt-based catalysts [9], where the dynamics could be studied in detail. Also for those experiments the subsecond time resolution of the new setup was essential. 


\section{CONCLUSIONS}

The new dedicated QEXAFS monochromator is a very valuable part of the SuperXAS beamline at the SLS, extending its possibilities to time resolved in situ studies. $\mathrm{Si}(111)$ and $\mathrm{Si}(311)$ crystals can be used, allowing covering the essential energy range of about 7 to $30 \mathrm{keV}$ with time resolutions of typically few seconds down to $20 \mathrm{~ms}$. The high data quality enables detailed observations of structural changes in the sample. The setup is convenient to use and well accepted by users of the SuperXAS beamline. The numerous scientific results obtained so far demonstrate the unique possibilities of the dedicated QEXAFS system at the SuperXAS Beamline at the SLS.

\section{REFERENCES}

1. R. Frahm, Nucl. Instr. Meth. Phys. Res. A 270, 578 (1988).

2. R. Frahm, Rev. Sci. Instrum. 60, 2515 (1989).

3. R. Frahm, B. Griesebock, M. Richwin and D. Lützenkirchen-Hecht, AIP Conf. Proc. 705, 1411 (2004).

4. R. Frahm, M. Richwin and D. Lützenkirchen-Hecht, Phys. Scr. T115, 974 (2005).

5. M. Nachtegaal, M. Harfouche, M. Willimann, R. Abela, T. Waterstradt, C. Venkataraman, M. Schwoerer-Böhning, W. Diete, K. Nurdan, J.A. van Bokhoven and R. Frahm, Proc. MEDSI 2008 / Pan-American SRI 2008, submitted for publication.

6. J. Stötzel, D. Lützenkirchen-Hecht, E. Fonda, N. De Oliveira, V. Briois and R. Frahm, Rev. Sci. Instrum. 79, 083107 (2008).

7. B.S. Clausen, H. Topsøe and R. Frahm, Advances in Catalysis 42, 315 (1998).

8. J. Singh, E.M.C. Alayon, M. Tromp, O.V. Safonova, P. Glatzel, M. Nachtegaal, R. Frahm and J.A. van Bokhoven, Angewandte Chemie International Edition 47, 9260 (2008).

9. J.-D. Grunwaldt, M.J. Beier, B. Kimmerle, A. Baiker, M. Nachtegaal, B. Griesebock, D. Lützenkirchen-Hecht, J. Stötzel and R. Frahm, Phys. Chem. Chem. Phys., DOI: 10.1039/b909872k (2009).

10. H. Bornebusch, B.S. Clausen, G. Steffensen, D. Lützenkirchen-Hecht and R. Frahm, J. Synchrotron Rad.. 6, 209 (1999).

11. J.-D. Grunwaldt, D. Lützenkirchen-Hecht, M. Richwin, S. Grundmann, B.S. Clausen and R. Frahm, J. Phys. Chem. B 105 $5161(2001)$.

12. J. Stötzel, D. Lützenkirchen-Hecht, R. Frahm, B. Kimmerle, A. Baiker, M. Nachtegaal, M.J. Beier and J.-D. Grunwaldt, Proc. XAFS14 Conference 2009, in press. 